\title{
Use of organoids in medicinal chemistry: challenges on ethics and biosecurity
}

\author{
Teresa Rinaldi*,1,2 \& Gianni Colotti*,3 \\ 1Department of Biology \& Biotechnology 'Charles Darwin' BBCD, La Sapienza University of Rome, 00185, Rome, Italy \\ ${ }^{2}$ Italian Army (Reserve), Ministry of Defence, 00100 , Rome, Italy \\ ${ }^{3}$ IBPM-CNR Institute of Molecular Biology \& Pathology, Italian National Research Council, 00185, Rome, Italy \\ *Authors for correspondence: teresa.rinaldi@uniroma1.it; gianni.colotti@uniroma1.it
}

First draft submitted: 20 July 2018; Accepted for publication: 25 February 2019; Published online: TBC

Keywords: 3D bioprinting $\bullet$ biosecurity $\bullet$ ethics $\bullet$ medicinal chemistry $\bullet$ medicine $\bullet$ organoids $\bullet$ pluripotent stem cells

\section{Organoids: applications}

Organoids are three-dimensional (3D) multicellular stem-cell-derived systems comprising organ-specific cell types, that exhibit key structural and functional properties of a variety of specific organs [1]; this technology has been chosen by Nature Methods as Method of the Year 2017 for the fascinating potential as tool to probe human biology and disease.

Organoids can be grown from pluripotent stem cells, embryonic stem cells, induced pluripotent stem cells and from organ-restricted adult stem cells. These cells differentiate in a controlled fashion in specific growth factors and culture media, with the support of a 3D matrix which allows cell growth, orientation and stratification. The resulting organoids replicate the development, metabolism, architecture and physiology of the real organ [2]. In fact, unlike in vitro traditional 2D cultures, organoids are formed by genomic stable, self-renewing and self-organizing stem cell-derived populations of differentiated cells that exhibit similar composition (they comprise all major cell lineages at frequencies similar to those in living tissue) and similar organ architecture and functionality as the tissue of origin. The technique allows expansions of tissue-specific stem cells and their differentiated progeny. Starting from very small amounts of material (such as biopsies); organoids can be expanded indefinitely, cryopreserved as biobanks and easily manipulated using techniques similar to those used for traditional 2D cell cultures [3]. Organoids are of course more physiologically relevant than traditional cell culture models and are easier to manipulate and study than in vivo mouse/human models. Gene-editing technologies can be used to produce and even self-transplant repaired, healthy organoids from patients with genetic defects.

In the last years, human organoids have been established for a wide range of organs, including salivary gland, esophagus, stomach, pancreas, liver, intestine, colon, kidney, prostate, lung, retina and brain. Using organoids, many types of human diseases have been modeled, ranging from Barrett's esophagus to Helicobacter pylori infection, cystic fibrosis, inflammatory bowel disease, bacterial or viral infections and even autism [3]. The luminal epithelia of human gastric organoids are efficiently colonized by Helicobacter pylori (the bacterium identified as the major cause of gastritis and one of the major agents of gastric cancer onset) and relevant physiological changes were demonstrated in this setting, including an increase in proliferation due to oncogenic CagA and increased $\beta$-catenin signaling [4]. Further, renal organoids have been used for the study of infections caused by Shiga-toxin-producing Escherichia coli [5]; these types of studies are yielding important contributions in understanding the pathogenic mechanisms of infections and are used to improve the strategies of treatment.

Many cancer models have been studied using organoids from glioblastoma, colorectal, gastrointestinal, pancreatic, prostate, breast, bladder and liver cancers [6]. Large patient-derived tumor organoid biobank collections from biopsy samples have been obtained, profiled and exploited for drug development and personalized cancer treatment purposes, and to analyze cancer-related processes and signaling pathways. Moreover, CRISPR/Cas9-mediated gene editing is used in organoids to evaluate candidate gene function in pathologies and tumorigenesis.

Organoids technology is also promising a boost to regenerative medicine. Transplantation of isogenic organoids from small patient biopsies or from allogenic but HLA-matched tissues represents a novel, exciting alternative to 
replace damage with healthy tissue by allogenic transplantation (at present limited by the scarce supply of healthy donor tissue and by tissue rejection issues).

Drug screening and testing in patient-derived organoids (PDOs) offer a novel and exciting alternative to traditional processes, at the edge between research and care, since on the one hand it provides insight into drug and disease mechanisms, and on the other hand it may become part of a treatment strategy for an individual patient (or a small cohort of patients).

The power and the possible controversies arising from the organoids approach can be understood with the possible selection of drug treatment for individual cystic fibrosis patients on the basis of an organoid swelling assay in intestinal organoids [7], or the patient-specific responses to specific drugs in biobanked colorectal and gastroesophageal cancer organoids from patients in Phase I/II clinical trials [8]. In the latter system, molecular profiling of tumoral PDOs was matched to drug-screening results, and responses to anticancer agents ex vivo in PDOs and PDO-based orthotopic mouse tumor xenograft models was compared with the responses of the patients in clinical trials; PDOs can recapitulate patient responses in the clinic, can define individual treatment responses and can be therefore used in personalized medicine programs [8].

The process of drug development itself may be profoundly changed by organoids technology; nowadays, Phase I clinical trials are aimed to determine the maximum tolerated dose of a novel drug molecule, while efficacy is evaluated in Phase II and III trials. Future early clinical-like studies to be performed in organoids may combine safety and efficacy determination [9], specifically targeting tumor cells while leaving healthy cells unharmed, resulting in reduced toxicities in patients. Hepatic organoid cultures provide a relevant model for preclinical testing of the hepatotoxicity of experimental compounds [10], while cardiac organoids can be used for testing cardiotoxicity [11], and kidney organoids have been used for toxicological studies [12].

Brain organoids are possibly the last frontier of this technique. Compared with $2 \mathrm{D}$ neural cell cultures, 3D organoids comprise up to millions of cells of more than one type, last longer (up to 2 years), mimic key features of developing brains structures and provide models for the study of autism, schizophrenia, Miller-Dieker syndrome and Zika-dependent microcephaly [13]. Some organoid models can be obtained by brain tissues removed from individuals during surgical procedures (for example, to treat seizures). Organoid mini-brains allow neuroscientists to study synaptic and other properties of neurons in intact brain circuits, to map the 3D morphology of circuits, to probe gene expression and to test compounds for the possible treatment of neurological disorders [13].

Organoids are therefore the basis for a revolution in several fields, since they are excellent model systems for a wide range of both basic research and translational applications and offer exciting possibilities in drug development and personalized medicine.

Nevertheless, there are some technical limits - self-assembled organoids still lack the microenvironment; a system of blood vessels and an immune system. Moreover, self-assembled organoids lack standardization and repeatability - an essential requirement to scale up this technology. In order to obtain standardization, organoids can be produced with 3D bioprinters; a robot that can move in three directions, equipped with an automated syringe that can dispense bio-ink with stem cells. With this technology, mini-organs of about $5 \mathrm{~mm}^{3}$ have already been obtained [14].

\section{Organoids \& ethics}

3D bioprinted organoids are revolutionizing the field of drug development and personalized medicine, organoid biobanks have already been created and there is a growing interest from pharmaceutical companies. Short-term ethical considerations rise for the application of organoids in disease modeling [15] who is the owner in the case of a tissue donation? Is the anonymity of the donor ensured? Are the donors fully aware that giving the informed consent to use their pluripotent stem cells, these could be used again in the future? Do they know the potential of this technology? Long-term ethical concerns may be an issue for organ transplantation in the future, since this technology goes beyond the fields of medical implants or tissue engineering. Is it ethical to implant a tissue or organ derived from nonhuman animals (pigs for instance) in humans [16]? A framework to fill this regulatory gap is necessary before the application of this technology spreads $[17,18]$.

Another important issue concerns the research on cerebral organoids: they exhibit neural connections and electrical activity, raising the question of whether they are, or possibly will be sentient. In principle, this can be measured with techniques already available, which are used for brain-injured noncommunicating patients. In case organoids will show some sensibility, an ethical discussion on their use in research, medicine and biotechnology would be necessary [19]. 


\section{Organoids \& biosecurity}

Further, there are many possible concerns about biosecurity - as it often happens for biological/medical sciences, the beneficial developments of organoids and 3D bioprinting (one of the technologies to produce organoids) would also involve an increasing risk that the same technologies could be used for harmful purposes (dual use concept) or for bioterrorism. In particular, in the wrong hands, lung and brain organoids could be used to test the toxicity of new chemical weapons/toxic chemicals or toxins, or to assess the infectivity of biological agents. These applications have already been tested with a lung on-a-chip to assess the ability of anthrax spores to infect a 'lung' grown from rabbit lung cells, and also to answer questions such as how many anthrax spores are necessary to cause the disease [20].

Concerns that 3D bioprinting and organoid technologies could lead to unregulated use is based on the flexibility of these model/organ systems, which are also attractive to researchers who are investigating dangerous pathogens, allowing them to reduce the numbers of animal tests.

Are the security restrictions in place able to cover these new technologies? Indeed, with access to the Internet and one thousand euros it is possible to buy mechanical, electrical and fluidic parts and assemble a 3D bioprinting mechanism - although accuracy remains an issue. Computer-aided design model data are also generally available on the Internet and bio-inks can be purchased in pharmacies, in supermarkets and from vendors of ingredients of molecular gastronomy. Fortunately, sophisticated skills are necessary to succeed in the production of organoids and the risk of diversion is low; nevertheless, raising awareness, in particular to Academia, is definitely a fundamental step to avoid the fall of this technology in the wrong hands.

\section{Financial \& competing interests disclosure}

The authors have no relevant affiliations or financial involvement with any organization or entity with a financial interest in or financial conflict with the subject matter or materials discussed in the manuscript. This includes employment, consultancies, honoraria, stock ownership or options, expert testimony, grants or patents received or pending, or royalties.

No writing assistance was utilized in the production of this manuscript.

\section{References}

1. Lancaster MA, Knoblich JA. Organogenesis in a dish: modeling development and disease using organoid technologies. Science 345(6194), 1247125 (2014).

2. Clevers H. Modeling development and disease with organoids. Cell 165(7), 1586-1597 (2016).

3. Fatehullah A, Tan SH, Barker N. Organoids as an in vitro model of human development and disease. Nat. Cell Biol. 18(3), 246-254 (2016).

4. Schlaermann $\mathrm{P}$, Toelle B, Berger $\mathrm{H}$ et al. A novel human gastric primary cell culture system for modelling Helicobacter pylori infection in vitro. Gut 65(2), 202-213 (2016).

5. DesRochers TM, Kimmerling EP, Jandhyala DM et al. Effects of Shiga toxin type 2 on a bioengineered three-dimensional model of human renal tissue. Infect. Immun. 83(1), 28-38 (2015).

6. Drost J, Clevers H. Organoids in cancer research. Nat. Rev. Cancer 18(7), 407-418 (2018).

7. Dekkers JF, Wiegerinck CL, de Jonge HR et al. A functional CFTR assay using primary cystic fibrosis intestinal organoids. Nat. Med. 19(7), 939-945 (2013).

8. Vlachogiannis G, Hedayat $S$, Vatsiou A et al. Patient-derived organoids model treatment response of metastatic gastrointestinal cancers. Science 359(6378), 920-926 (2018).

9. Habets MG, van Delden JJ, Niemansburg SL, Atkins HL, Bredenoord AL. One size fits all?: ethical considerations for examining efficacy in first-in-human pluripotent stem cell studies. Mol. Ther. 24(12), 2039-2042 (2016).

10. Huch M, Gehart H, van Boxtel R et al. Long-term culture of genome-stable bipotent stem cells from adult human liver. Cell 160(1-2), 299-312 (2015).

11. Eder A, Vollert I, Hansen A, Eschenhagen T. Human engineered heart tissue as a model system for drug testing. Adv. Drug. Deliv. Rev. 96, 214-224 (2016).

12. Takasato M, Er PX, Chiu HS et al. Kidney organoids from human iPS cells contain multiple lineages and model human nephrogenesis. Nature 526(7574), 564-568 (2015).

13. Farahany N, Greely HT, Hyman S et al. The ethics of experimenting with human brain tissue. Nature 556(7702), 429-432 (2018).

14. Wang X, Ao Q, Tian X et al. 3D bioprinting technologies for hard tissue and organ engineering. Materials 9(10), 802 (2016).

15. Gilbert F, O'Connell CD, Mladenovska T, Dodds S. Print me an organ? Ethical and regulatory issues emerging from 3D bioprinting in medicine. Sci. Eng. Ethics 24(1), 73-91 (2018). 
16. Ravnic DJ, Leberfinger AN, Koduru SV et al. Transplantation of bioprinted tissues and organs: technical and clinical challenges and future perspectives. Ann. Surg. 266(1), 48-58 (2017).

17. Wolinsky H. Printing organs cell-by-cell: 3-D printing is growing in popularity, but how should we regulate the application of this new technology to health care?. EMBO Rep. e201439207 (2014).

18. Patuzzo S, Goracci G, Gasperini L, Ciliberti R. 3D bioprinting technology: scientific aspects and ethical issues. Sci. Eng. Ethics 24(2), 335-348 (2018).

19. Lavazza A, Massimini M. Cerebral organoids: ethical issues and consciousness assessment. J. Med. Ethics 44(9), 606-610 (2018).

20. Powell JD, Hutchison JR, Hess BM, Straub TM. Bacillus anthracis spores germinate extracellularly at air-liquid interface in an in vitro lung model under serum-free conditions. J. Appl. Microbiol. 119(3), 711-723 (2015). 